\title{
BMJ Open Smoking cessation interventions for people living in rural and remote areas: a systematic review protocol
}

\author{
Alistair Lum, Eliza Skelton, Kristen Louise McCarter (D) , Tonelle Handley, \\ Lucy Judd, Billie Bonevski
}

To cite: Lum A, Skelton E, McCarter KL, et al. Smoking cessation interventions for people living in rural and remote areas: a systematic review protocol. BMJ Open 2020;10:e041011. doi:10.1136/ bmjopen-2020-041011

- Prepublication history for this paper is available online. To view these files, please visit the journal online (http://dx.doi. org/10.1136/bmjopen-2020041011).

Received 01 June 2020 Revised 12 October 2020 Accepted 30 0ctober 2020

D) Check for updates

(c) Author(s) (or their employer(s)) 2020. Re-use permitted under CC BY-NC. No commercial re-use. See rights and permissions. Published by BMJ.

School of Medicine \& Public Health, University of Newcastle, Callaghan, NSW, Australia

Correspondence to

Dr Alistair Lum;

alistair.lum@newcastle.edu.au

\section{ABSTRACT}

Introduction Smoking rates among people living in rural and remote areas are higher and quit rates are lower over the past 10 years compared with people living in suburban and urban areas. Higher smoking rates contribute to greater tobacco-related disease and morbidity in rural and remote areas. Physical and social isolation, greater exposure to pro-tobacco marketing, pro-tobacco social norms, and lower socioeconomic and educational levels are contributing to these higher smoking rates and lower quit rates. Smoking cessation interventions for people in rural and remote areas have been conducted, however little is known about their effectiveness or their mechanisms of action as well as the quality of such research. Behaviour change techniques (BCTs) are mechanisms of action derived from behaviour change theory, such as goal setting and reward. Improved understanding of the contribution of BCTs for smoking cessation in the rural and remote population will support future intervention development. We aim to review the literature on smoking cessation interventions for people living in rural and remote areas to inform evidence about intervention effectiveness and mechanisms of action. Methods and analysis We will conduct a systematic review using seven scientific databases (EMBASE, MedLine, PsycINFO, CINAHL, Cochrane, Informit Health and Scopus). We will include peer-reviewed journal articles published in English that examine a smoking cessation intervention delivered to people living in rural and remote areas in the USA, Canada and Australia. We will examine outcome data relating to intervention effectiveness (eg, point prevalence abstinence or continuous abstinence), as well as the BCTs used in included interventions and their relationship with intervention outcomes. We will also assess the feasibility, acceptability and quality of research interventions of included articles, and provide graded recommendations based on the review outcomes. Data will be synthesised using narrative approaches and interpreted using content analysis.

Ethics and dissemination Ethics was not required for this systematic review. The results will be disseminated through peer-reviewed publication and at conferences by presentations.

PROSPERO registration number 177398.

\section{INTRODUCTION}

Rates of smoking in high-income countries are falling worldwide. Smoking rates have more than halved in Australia, Canada and the USA between 1990 and 2015. ${ }^{1}$ Between

\section{Strengths and limitations of this study}

- This systematic review will provide guidance to support tailored smoking cessation interventions for people living in rural and remote communities.

- This review has been registered with PROSPERO which increases the transparency of its methods and decreases reporting bias.

- By identifying the behaviour change techniques (BCTs) associated with intervention effectiveness, this review will provide greater clarity about what intervention features might lead to the greatest improvement in smoking cessation rates in this unique population.

- The identification of BCTs will rely on the authors of included studies to provide their intervention manuals, therefore non-compliance to this request may limit the quality of this review.

$14 \%$ and $16 \%$ of adults in Australia, Canada and the USA smoke tobacco. ${ }^{2-4}$ The decline in smoking rates in these countries appears to be less apparent among people living in rural areas when compared with urban areas. One study has shown that the decline in smoking rates among people in urban areas in the USA was $2 \%$ between 2007 and 2014, yet was $0 \%$ for those in rural regions. ${ }^{5}$ This small but significant difference in rates of smoking cessation accounted for smoking rates that were 1.3 times higher among the rural population than the urban population. People living in rural areas of Australia are similarly 1.3 times more likely to smoke tobacco than those in urban areas. ${ }^{6}$ The difference in Canada between the two geographically separated populations is smaller but still significant, with rural residents $6 \%$ more likely to smoke than urban residents. ${ }^{7}$

Tobacco is a primary cause of preventable death and disease in high-income coun$\operatorname{tries}^{8}$ and the key modifiable risk factor for the development of cardiovascular disease, lower respiratory infections, chronic obstructive pulmonary disease (COPD) and cancer. ${ }^{9}$ 
Rates of tobacco-associated cancer incidence in the USA fell by $3.4 \%$ in rural residents, a significantly smaller decline than the $13.8 \%$ among urban residents. ${ }^{10}$ The prevalence of heart disease is higher among people living in rural areas of the USA than urban areas, and cigarette smoking appears to be partially responsible for this difference. ${ }^{11}$ Rural residents in the USA are expected to live 2 years less than urban residents, with the tobacco-related diseases such as cardiovascular diseases, COPD and lung cancers acting as the primary contributors to early mortality. ${ }^{12}$ Children living in rural areas are also more likely to be exposed to secondhand smoke than their urban counterparts, which also contributes to poorer health outcomes. ${ }^{13}$

Numerous factors may be perpetuating higher smoking rates among people in rural and remote areas. Tobacco farming in rural areas of the USA is linked to higher smoking rates in these areas compared with rural areas not associated with tobacco farming and urban areas. ${ }^{14}$ Rural Australian Aboriginal women have described smoking as a social norm that fosters belonging. ${ }^{15}$ First Nations people in Canada, the USA and Australia are both more likely to smoke than non-First Nations people and more likely to live in rural towns. ${ }^{16-19}$ A 2019 review indicated that rural residents in the USA were exposed to greater pro-tobacco advertising that appealed to cultural standards within rural communities. ${ }^{20}$ Antitobacco media campaigns, especially localised messages on print, were reportedly received by rural communities with high reception. ${ }^{20}$ Limited access to healthcare may also contribute to fewer opportunities for professional advice and support to quit smoking. ${ }^{21}$ Lower educational levels among rural and remote residents compared with urban counterparts may also help maintain health beliefs supporting tobacco use, while lower socioeconomic status may reduce access to smoking cessation medication and support. ${ }^{721}$

Australia, Canada and the USA all have strong smoking reduction goals, within which each country has specified the need to target subpopulations who smoke at higher rates and experience higher tobacco-related harm than the general population. People living in rural and remote areas are currently experiencing such disparities, and require specialised support to quit smoking. People who smoke and live in rural and remote areas have recommended that tailored smoking cessation interventions should be designed as a 'non-judgemental partnership' approach involving physicians. ${ }^{22}$ Australian rural smokers may prefer greater steps taken from the primary healthcare system. ${ }^{23}$ These steps include appointing dedicated tobacco staff, collaborating with general practitioners and mental health services from the start of cessation trials and any service that makes it financially easier or more convenient to participate in trials.

To identify the active ingredients of smoking cessation interventions, Michie et $a l^{24}$ developed the behaviour change technique (BCT) taxonomy. BCTs refer to explicitly described components of interventions that can modify behaviour, which are organised into four functions: directly addressing motivation, maximising self-regulatory capacity or skills, promoting adjuvant activities and supporting other BCTs. Forty-three BCTs have been identified in smoking cessation interventions, with 17 linked to improved short-term ( $<6$ months) smoking cessation outcomes. ${ }^{25}$ The 17 BCTs linked to smoking cessation included strengthening identity as an ex-smoker and providing rewards contingent on quitting smoking (directly addressing motivation), advising on changing routine and facilitating relapse prevention and coping strategies (maximising self-regulatory capacity or skills), giving options for additional support and asking about experiences of using stop-smoking medications (promoting adjuvant activities), and eliciting client's views (supporting other BCTs).

Research has also explored BCTs for smoking cessation among target populations, such as pregnant women. ${ }^{26}$ Interventions targeted at pregnant women appeared to be more likely to quit smoking when interventions included the BCTs of advising on or facilitating social support and providing rewards for quitting smoking. These two BCTs were unique to the target population (ie, were not linked to outcome change among the generic population), highlighting the importance of tailoring intervention components to the population of interest. However, a review of 17 smoking interventions targeting people in low-income groups which identified 11 BCTs found no evidence that BCTs were related to intervention effectiveness. ${ }^{27}$

Smoking cessation interventions designed to meet the unique needs of people living in rural and remote areas have been developed. As yet, it is unclear whether interventions with this focus have been effective at helping people quit smoking, and which BCTs have contributed to intervention effectiveness. We aim to systematically review the literature examining smoking cessation interventions for people living in rural and remote areas of Australia, Canada and the USA to better understand their effectiveness and mechanisms of action. The objective of this review is to develop a strong understanding of the available targeted support to provide clearer direction for future development and implementation of smoking cessation interventions for people living in rural and remote areas of Australia, Canada and the USA. Further, no known studies have examined the inclusion of BCTs in rural or remote smoking cessation interventions. Identifying BCTS potentially related to more effective interventions will hopefully improve future tailored smoking cessation interventions for this priority population.

Our focus on Australia, Canada and the USA is based on the fact that each country is high income, has a similar geographical size, has similar smoking rates and has similar smoking cessation objectives. While many similarities exist, some differences should be mentioned. Canada and the USA have similar rural population percentages (18.9\% and $19.3 \%$, respectively), and Australia has a slightly larger rural population percentage (29.0\%). Australia and Canada have similar populations and 
population densities, while the USA has a much larger population. With consideration of the differences between countries, the similarities will help generalise the findings across countries.

It is clear that there is a need for improved smoking cessation interventions that are tailored to the unique needs of people living in rural and remote areas to help reduce smoking rates and tobacco-related diseases. Therefore, we aimed to review the effectiveness of smoking cessation interventions targeting people who live in rural and remote areas of Australia, Canada and the USA. We will focus on examining BCTs to help identify the primary mechanisms of action behind effective interventions so that future interventions can be designed specifically for people from rural and remote areas.

We asked two primary research questions:

1 . What is the effectiveness of smoking cessation interventions targeting people who live in rural and remote areas of Australia, Canada and the USA?

2. How many and what types of BCTs are employed in smoking cessation interventions, and are these related to intervention effectiveness?

We also asked two secondary research questions:

1. What is the feasibility and acceptability of smoking cessation interventions targeting people who live in rural and remote areas of Australia, Canada and the USA?

2. What is the quality of evidence in research examining smoking cessation interventions among people who live in rural and remote areas of Australia, Canada and the USA?

\section{METHODS}

\section{Study design}

We will complete a systematic review examining effectiveness of smoking cessation interventions for people living in rural and remote regions. We will follow the Preferred Reporting Items for Systematic Reviews and Meta-Analyses guidelines during all parts of the review. ${ }^{28}$ Any protocol amendments will be registered to PROSPERO and will be indicated in the review publication.

\section{Eligibility criteria}

\section{Study designs}

We will include randomised controlled/clinical trials, pilot studies, pre-post intervention studies or feasibility studies. All included articles must include an intervention. Control or comparison groups of any kind (eg, usual care, urban population) may be included, however, it is not mandatory that the study design include a control or comparison group. Studies that only include observational data, review studies and case studies will be excluded.

\section{Participants}

Participants must be classified as tobacco smokers who live in a rural or remote region of Australia, Canada and the USA. Tobacco smokers must be defined by the minimum of smoking combustible tobacco (eg, cigarette, cigars or pipes) and smoking multiple days per week. Studies including tobacco users (eg, smokeless tobacco) will be included provided that smokeless tobacco users do not account for more than $10 \%$ of the participant group. Studies examining e-cigarettes will be excluded. Rural and remote may be defined as physical distance and/or isolation or by number of people living in the area. Due to differences between countries and within countries across time, we are reliant on original study authors to accurately classify participants as rural or remote. Studies including participants from non-rural or remote areas may be included provided that the outcomes for rural and remote participants are presented separately to urban or suburban participants.

\section{Interventions}

Interventions targeting smoking cessation and/or reduction will be included in this systematic review. Interventions may be pharmacological (eg, nicotine replacement therapy, varenicline, bupropion), cognitive and behavioural therapies (eg, motivational interviewing, cognitive-behavioural therapy, contingency management, self-help, support groups), or a combination of both. We will exclude research articles including interventions that use public health (eg, media campaigns, public policies) or are clinical (eg, phase I-IV trials) as reviewing these interventions is beyond the scope of this review.

\section{Outcomes}

The primary outcome we will examine is the effectiveness of the intervention in terms of smoking cessation (as measured by continuous abstinence, prolonged abstinence and point prevalence abstinence at any time frame) and/or smoking reduction (as measured by reduction in number of cigarettes per day). We will also explore outcomes associated with BCTs (ie, types, numbers of, relationship with intervention effectiveness), feasibility and acceptability of the intervention, and the quality of included studies.

\section{Additional eligibility criteria}

We will only include articles that are published in English and that have been published in a peer-reviewed journal. We will exclude published abstracts, conference abstracts and dissertations. No publication dates will be specified.

\section{Search strategy}

We will search seven databases: EMBASE, MedLine, PsycINFO, CINAHL, Informit Health, Cochrane and Scopus. We will use keywords consistent across all databases and Medical Subject Headings applicable to specific databases. The search terms are outlined in table 1 and are presented by the overarching theme relevant to our research questions: Rural and remote populations; Tobacco smoking cessation; Intervention and Country of interest. The search terms within each group will be joined by OR and the themes will be combined using AND. The search terms have been developed with guidance from the Cochrane Database Tobacco Addiction 


\begin{tabular}{|c|c|}
\hline Theme & Search terms \\
\hline $\begin{array}{l}\text { Rural and } \\
\text { remote } \\
\text { population }\end{array}$ & $\begin{array}{l}\text { Rural* OR Remote* OR Isolated* or Rural } \\
\text { Population/ Relevant database MeSH terms }\end{array}$ \\
\hline \multirow[t]{2}{*}{$\begin{array}{l}\text { Tobacco } \\
\text { smoking } \\
\text { cessation }\end{array}$} & 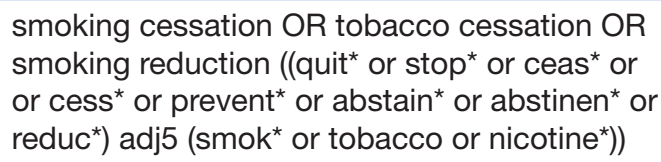 \\
\hline & Relevant database MeSH terms \\
\hline \multirow[t]{2}{*}{ Intervention } & Trial* OR Intervention* OR Pilot* OR Program* \\
\hline & Relevant database MeSH terms \\
\hline \multirow[t]{2}{*}{$\begin{array}{l}\text { Countries of } \\
\text { interest }\end{array}$} & $\begin{array}{l}\text { Australia* OR Canad* OR United States OR } \\
\text { USA OR America* }\end{array}$ \\
\hline & Relevant database MeSH terms \\
\hline
\end{tabular}

*refers to truncations.

$\mathrm{MeSH}$, Medical Subject Headings.

Group search terms (https://tobacco.cochrane.org/ resources/cochrane-tag-specialised-register).

\section{Screening}

One author will screen all titles and abstracts. Another two authors will each review $50 \%$ of titles and abstracts so that each title and abstract is reviewed by two authors. Any disagreements will be discussed between all three authors who are screening. If a resolution cannot be found, the six authors named as investigators will hold a discussion until a resolution is found.

One author will screen all publications that were not excluded by title or abstract. Another two authors will each review $50 \%$ of the publications, so that each full text is reviewed by two authors. Any disagreements will be discussed between all three screening authors. If a resolution cannot be found, the six authors named as investigators will hold a discussion until a resolution is found. Titles, abstracts and full texts will be screened using the Covidence software V.1919 73d6c782.

\section{Data extraction}

The team will develop a data extraction form based on the aims of the study. Outcome data will include study characteristics (eg, year completed, design, country of study, sample size), participant characteristics (eg, age, sex, education, employment, income, race/ethnicity, rurality), smoking characteristics (eg, number of cigarettes smoked daily, age smoking commenced, tool to assess smoking status and nicotine dependence), intervention characteristics (eg, pharmacological, non-pharmacological, length, setting, BCTs), intervention effectiveness (eg, change in smoking behaviours, change in smoking cravings) and feasibility/acceptability outcomes (eg, recruitment and retention rate, participant satisfaction). One author will extract data from all included publications. Another two authors will extract data from $50 \%$ of included manuscripts. Any disagreements will be discussed between all three screening authors. If a resolution cannot be found, the six authors named as investigators will hold a discussion until a resolution is found. BCTs will be extracted by one author with training in identifying BCTs in smoking cessation research using the BCT taxonomy. ${ }^{24}$ Data will be extracted using the Covidence software.

\section{Quality assessment}

We will assess risk of bias at the study level using tools developed by the Scottish Intercollegiate Guidelines Network (SIGN). SIGN has developed multiple tools specific for different study designs, including one for controlled trials, ${ }^{29}$ cohort $_{\text {studies }}{ }^{30}$ and case-control studies. Using the SIGN guidelines will allow for assessment of different study designs using tools developed with a similar approach. The tools judge studies as high quality, acceptable, low quality or unacceptable. We will not exclude articles from review based on their score on the quality appraisal tool, but will use the scores to improve our understanding of the quality of the trial and reliability of the findings.

We will use the Grading of Recommendations Assessment, Development and Evaluation (GRADE) system to assess the quality of evidence presented by original research included in the review. ${ }^{31}$ The GRADE tool bases quality of evidence on the methodological quality, risk of bias, inconsistencies, imprecisions and indirectness of pooled research for outcomes prespecified by the research team. We will report the strength of recommendations as determined by quality of evidence and the perceived risk-benefit ratio of the recommendations.

One author will conduct risk of bias assessment in $100 \%$ of included articles and two authors will assess $50 \%$ of the included articles for risk of bias. Any disagreements will be discussed between all authors completing quality appraisal. If a resolution cannot be found, the six authors named as investigators will hold a discussion until a resolution is found.

One author will assess the quality of evidence using the GRADE tool and will present the findings to the investigator team. The team will discuss the assessment and modify the reported strength of recommendations.

\section{Data synthesis}

We will analyse data using a narrative approach (ie, interpreting data using qualitative, rather than quantitative methods) to account for the anticipated heterogeneity of interventions and outcome measures. ${ }^{32}$ We will use content analysis techniques to examine trends in outcomes and in the role of BCTs. ${ }^{33}$ Codes will be based on statistical outcomes (eg, p values) and BCTs. We will examine the relationship between BCTs and statistical outcomes within studies and examine trends in these relationships across studies. We will interpret and report the data accordingly.

We are not planning on analysing according to groups or subsets; however, we will analyse subgroups or subsets 
if it becomes apparent that they are relevant to the outcomes.

\section{Patient and public involvement \\ No patient involved.}

\section{Study status}

At the time of submission of this protocol, the authors had completed screening of titles, abstracts and full texts, and were beginning data extraction and quality assessment.

\section{DISCUSSION}

People living in rural and remote areas of Australia, Canada and the USA smoke at higher rates than their urban counterparts, are less likely to quit and are more likely to face the adverse health consequences of smoking. ${ }^{5-7}$ Rural and remote living is associated with unique challenges beyond physical and social isolation, including greater exposure to pro-tobacco marketing, living within a setting where smoking is a social norm, and lower education and socioeconomic levels. ${ }^{15} 2021$ Smoking cessation interventions are being delivered to people living in rural and remote areas, however little is known about their effectiveness and what mechanisms are leading to the best outcomes. Without tailored interventions targeting the unique needs associated with rural and remote living, current interventions may be doing little to reduce smoking rates and improve health in this priority population.

This systematic review will take a comprehensive approach to examine the effectiveness of smoking cessation interventions as well as the intervention characteristics that are most beneficial for people living in rural and remote areas. By identifying the BCTs associated with intervention effectiveness, this review will provide greater clarity about what intervention features might lead to the greatest reduction in smoking in this unique population. This knowledge will support researchers develop evidence-based interventions.

This review will also assess feasibility and acceptability outcomes and the quality of published articles. We will be able to provide a clear analysis of the state of smoking cessation research targeting people living in rural and remote areas, as this group is often identified as an underserved population. It is anticipated that our research will not only identify some factors contributing to optimal intervention outcomes, but also that more research is needed to meet the needs of rural and remote population.

Contributors AL and ES prepared the systematic review protocol and drafted the manuscript. All authors, including AL, ES, KLM, TH, LJ and BB, provided feedback on the protocol and manuscript, and read and approved the final manuscript.

Funding This systematic review was funded by the Hunter Cancer Research Alliance Implementation Science and Impact Flagship Grant (GS190025). The funding was to conduct a systematic review.

Disclaimer The funding body had no other role in the collection, analysis or interpretation of the data.

Competing interests None declared.
Patient and public involvement Patients and/or the public were not involved in the design, or conduct, or reporting, or dissemination plans of this research.

Patient consent for publication Not required.

Provenance and peer review Not commissioned; externally peer reviewed.

Open access This is an open access article distributed in accordance with the Creative Commons Attribution Non Commercial (CC BY-NC 4.0) license, which permits others to distribute, remix, adapt, build upon this work non-commercially, and license their derivative works on different terms, provided the original work is properly cited, appropriate credit is given, any changes made indicated, and the use is non-commercial. See: http://creativecommons.org/licenses/by-nc/4.0/.

ORCID iD

Kristen Louise McCarter http://orcid.org/0000-0002-2638-6381

\section{REFERENCES}

1 Reitsma MB, Fullman N, Ng M, et al. Smoking prevalence and attributable disease burden in 195 countries and territories, 19902015: a systematic analysis from the global burden of disease study 2015. Lancet 2017;389:1885-906.

2 Scoll M, Winstanley M. Tobacco in Australia: facts and issues. Melbourne: Cancer Council Victoria, 2019.

3 Centers for Disease Control Prevention. Current cigarette smoking among adults in the United States. Centers for Disease Control and Prevention, 2019.

4 Statistics Canada. Smoking, 2018. Ottowa: Statistics Canada., 2018

5 Doogan NJ, Roberts ME, Wewers ME, et al. A growing geographic disparity: rural and urban cigarette smoking trends in the United States. Prev Med 2017;104:79-85.

6 Australian Institute of Health Welfare. Risk factors to health. Canberra: AlHW, 2017.

7 Li FX, Robson PJ, Ashbury FD, et al. Smoking frequency, prevalence and trends, and their socio-demographic associations in Alberta, Canada. Can J Public Health 2009;100:453-8.

8 Goodchild M, Nargis N, Tursan d'Espaignet E. Global economic cost of smoking-attributable diseases. Tob Control 2018;27:58-64.

9 Thun MJ, Carter BD, Feskanich D, et al. 50-year trends in smoking-related mortality in the United States. N Engl J Med 2013;368:351-64.

10 Zahnd WE, James AS, Jenkins WD, et al. Rural-urban differences in cancer incidence and trends in the United States. AACR, 2018.

11 O'Connor A, Wellenius G. Rural-urban disparities in the prevalence of diabetes and coronary heart disease. Public Health 2012;126:813-20.

12 Singh GK, Siahpush M. Widening rural-urban disparities in life expectancy, U.S., 1969-2009. Am J Prev Med 2014;46:e19-29.

13 Singh GK, Siahpush M, Kogan MD. Disparities in children's exposure to environmental tobacco smoke in the United States, 2007. Pediatrics 2010;126:4-13.

14 Butler KM, Hedgecock S, Record RA, et al. An evidence-based cessation strategy using rural smokers' experiences with tobacco. Nurs Clin North Am 2012;47:31-43.

15 Passey ME, Gale JT, Sanson-Fisher RW. "It's almost expected": rural Australian Aboriginal women's reflections on smoking initiation and maintenance: a qualitative study. BMC Womens Health 2011;11:55.

16 Statistics ABo. National aboriginal and torres strait Islander social survey, 2014-15. Australian Bureau of statistics Canberra, 2016.

17 First Nations Information Governance Centre. First nations regional health survey (Rhs) 2008/10: national report on adults youth and children living in first nations communities, 2012.

18 Substance Use and Mental Health Services Administration. Results from the 2016 national survey on drug use and health: detailed tables. Rockville, MD: Center for Behavioral Health Statistics and Quality, 2017.

19 Dewees S, Marks B. Twice invisible: understanding rural native America. Albuquerque, NM: First Nations Development Institute, 2017.

20 Cruz TB, Rose SW, Lienemann BA, et al. Pro-tobacco marketing and anti-tobacco campaigns aimed at vulnerable populations: a review of the literature. Tob Induc Dis 2019;17:68.

21 Jones CA. Health status and health care access of farm and rural populations. DIANE Publishing, 2009.

22 Hutcheson TD, Greiner KA, Ellerbeck EF, et al. Understanding smoking cessation in rural communities. $J$ Rural Health 2008;24:116-24.

23 Tall JA, Brew BK, Saurman E, et al. Implementing an anti-smoking program in rural-remote communities: challenges and strategies. Rural Remote Health 2015;15:3516. 
24 Michie S, Hyder N, Walia A, et al. Development of a taxonomy of behaviour change techniques used in individual behavioural support for smoking cessation. Addict Behav 2011;36:315-9.

25 West R, Walia A, Hyder N, et al. Behavior change techniques used by the English stop smoking services and their associations with shortterm quit outcomes. Nicotine Tob Res 2010;12:742-7.

26 Lorencatto F, West R, Michie S. Specifying evidence-based behavior change techniques to aid smoking cessation in pregnancy. Nicotine Tob Res 2012;14:1019-26.

27 Bull ER, McCleary N, Li X, et al. Interventions to promote healthy eating, physical activity and smoking in low-income groups: a systematic review with meta-analysis of behavior change techniques and delivery/context. Int J Behav Med 2018;25:605-16.
28 Moher D, Liberati A, Tetzlaff J, et al. Preferred reporting items for systematic reviews and meta-analyses: the PRISMA statement. Ann Intern Med 2009;151:264-9.

29 Scottish Intercollegiate Guidelines Network. Methodology checklist 2: controlled trials, 2014

30 Scottish Intercollegiate Guidelines Network. Methodology checklist 3 : cohort studies, 2014

31 Guyatt GH, Oxman AD, Schünemann HJ, et al. Grade guidelines: a new series of articles in the Journal of clinical epidemiology. J Clin Epidemiol 2011;64:380-2.

32 Popay J, Roberts H, Sowden A. Guidance on the conduct of narrative synthesis in systematic reviews. A product from the ESRC methods programme version, 2006.

33 Elo S, Kyngäs $\mathrm{H}$. The qualitative content analysis process. J Adv Nurs 2008;62:107-15. 